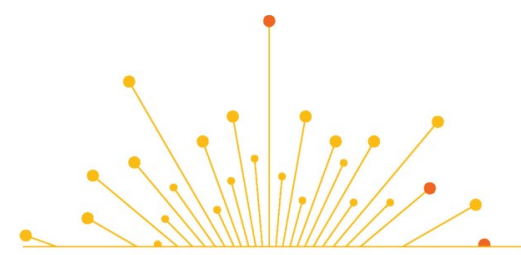

SCIENCE TABLE

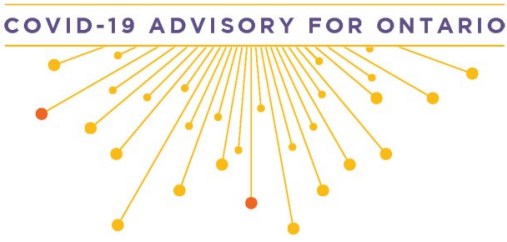

Version 1.0

Published: August 12, 2020

Citation: Watts T, Yoojin C, Odutayo A, et al. Decrease in Antibodies Against SARS-CoV-2 Over Time and Current Role of Serological Testing. Science Briefs of the Ontario COVID19 Science Advisory Table. 2020;1(2). https:// doi.org/10.47326/ocsat.2020.01.02.1.0

Author Affiliations: The affiliations of the members of the Ontario COVID-19 Science Advisory Table can be found at www.covid19 -sciencetable.ca.

Declarations of Interest: The declarations of interest of the members of the Ontario COVID-19 Science Advisory Table can be found at www.covid19-sciencetable.ca.

About Us: The Ontario COVID-19 Science Advisory Table is a group of scientific experts and health system leaders who evaluate and report on emerging evidence relevant to the COVID-19 pandemic, to inform Ontario's response. Our mandate is to provide weekly summaries of relevant scientific evidence for the COVID-19 Health Command Table of the Province of Ontario, integrating information from existing scientific tables, Ontario's universities and agencies, and the best global evidence. The Science Table summarizes its findings for the Health Command Table and the public in Science Briefs.

Correspondence to: Secretariat of the Ontario COVID-19 Science Advisory Table (info@covid19-sciencetable.ca)

Copyright: 2020 Ontario COVID-19 Science Advisory Table. This is an open access document distributed under the terms of the Creative Commons Attribution License, which permits unrestricted use, distribution, and reproduction in any medium, provided that the original work is properly cited.

\section{Decrease in Antibodies Against SARS-CoV-2 Over Time and Current Role of Serological Testing}

Tania Watts, Yoojin Choi, Ayodele Odutayo, Gerald A. Evans, David N. Fisman, Allison McGeer, Samira Mubareka, Samir N. Patel, Peter Jüni on behalf of the Ontario COVID-19 Science Advisory Table

\section{Key Message}

The extent to which people after an infection with SARS-CoV-2 are protected from re-infection is unknown. The observed decrease in antibodies against SARS-CoV-2 over time does not mean that immunity against SARS-CoV-2 is lost after infection. Serological testing for the presence of SARS-CoV-2 specific antibodies currently does not provide meaningful clinical information for the individual.

\section{Summary}

\section{Background}

There has been much discussion about whether infection with SARS-CoV-2 results in immunity to future infections.

After a viral infection, the immune system recognizes specific antigens of the virus, responds to the virus and can remember it. Memory $B$ cells and memory T cells are white blood cells that persist after the initial infection to allow a rapid response to a repeat infection, usually allowing rapid elimination of the infectious agent without the development of disease. B cells are involved in the antibody response. T cells support B cells in generating a strong and persistent antibody response and are involved in a cell driven immune response to eliminate cells that have already been infected. After some infections or vaccines, antibody responses can persist for a long time, and, if the antibodies are neutralizing, they can completely prevent re-infection with the same agent.

The majority of people infected with SARS-CoV-2 (the virus that causes COVID-19) develop specific antibodies and T cells against the virus, but the immune response may vary by the clinical severity of infection. To date, we do not know the specific type or amount of immune response that is sufficient to protect from reinfection with SARS-CoV-2.

The duration of the antibody response varies as well. In a recently published study, $81 \%$ of infected asymptomatic individuals and $62 \%$ of symptomatic individuals showed a decrease in the levels of neutralizing antibodies 8 weeks after hospital discharge, which is shorter than what was found for other coronaviruses. This led to concerns that those who have been infected with SARS-CoV-2 might not be immune to it.

\section{Questions}

What is the clinical importance of the recently reported decrease in antibodies 
against SARS-CoV-2 over time?

What is the current role of serological testing for antibodies against SARS-CoV-2?

\section{Findings}

Adaptive immunity against SARS-CoV-2 is not only based on antibodies, but also includes a T-cell response. A recent study found that 15 out of 18 COVID-19 patients developed specific T-cell responses against SARS-CoV-2.

There is evidence of both memory B cells and memory T cells against SARS-CoV-2 in blood samples of people who experienced an infection with SARS-CoV-2. These memory cells are likely to stimulate a rapid immune response in case of a re-infection.

Some people who have never been exposed to SARS-CoV-2 may have already acquired adaptive cellular immunity, with specific T-cell responses against SARS-CoV-2. These T-cell responses are likely due to previous infections with other coronaviruses, such as those causing the common cold.

\section{Interpretation}

Antibody responses appear less pronounced in infected asymptomatic individuals than in symptomatic individuals, and antibody levels appear to decrease relatively fast after an infection with SARS-CoV-2, but the clinical importance of these observations is unknown.

The detection of both memory $T$ cells and memory $B$ cells from recovered individuals suggests the potential for the adaptive immune system to respond quickly upon a re-infection.

The immune response to SARS-CoV-2 and the factors important for protection against re-infection are incompletely understood. Therefore, testing for the presence of SARS-CoV-2 specific antibodies currently does not provide any meaningful clinical information to the individual being tested. Serological testing should therefore be limited to research and public health use only.

The adaptive immune response to SARS-CoV-2 discussed in this report only reflects what is observed in a natural infection. The issues described in this report should not be used to predict the potential effects of future vaccines.

\section{Background}

There has been much discussion about whether infection with SARS-CoV-2 results in immunity to future infections.

After a viral infection, the immune system recognizes specific antigens of the virus, responds to the virus, and can remember it. $B$ cells are involved in the antibody response. $T$ cells support $B$ cells in generating a strong and persistent antibody response and are involved in a cell driven immune response to eliminate cells that have been infected (Figure). ${ }^{1}$

Memory B cells and memory T cells are white blood cells that persist after the initial infection to allow a rapid response to a repeat infection, usually allowing rapid elimination of the infectious agent without the development of disease. Memory B cells and memory $T$ cells recognize the antigens of a pathogen, such as SARS-CoV-2 (the virus that causes COVID-19), if an individual is infected more than once. The memory cells can trigger antibody-mediated and cell-mediated adaptive immune responses that are typically more efficient than the response mounted when the individual was infected for the first time. ${ }^{1}$ 
Another important part of protective immunity is the persistence of antibodies due to the presence of long-lived plasma cells, which constitutively produce antibodies over a prolonged period of time (Figure). ${ }^{2}$

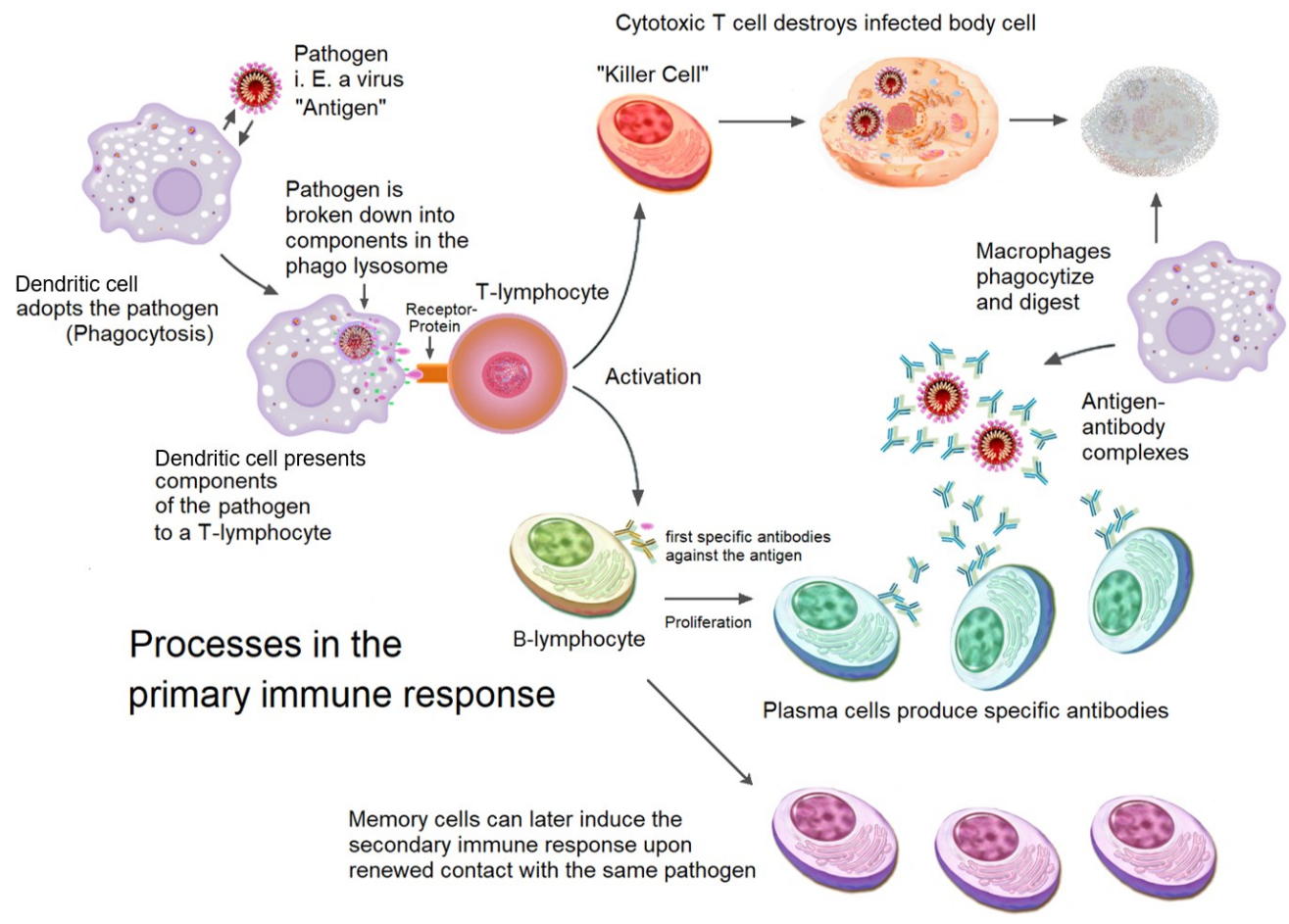

Figure. Simplified overview of adaptive immune response

Immune cells called Dendritic cells survey the body and can detect a new viral infection. Dendritic cells ingest the viral particles, break them down and present components of the virus as a signal to $T$ cells. $T$ cells that receive these signals can either kill cells that are infected with the same virus (top), or help B cells become plasma cells to produce antibodies against the virus (bottom). After cell-mediated and/or antibody-mediated clearance of the virus, memory $T$ cells, memory $B$ cells and long-lived plasma cells can remain in the body for decades. Antibodies from long-lived plasma cells can provide immediate protection in case of a re-infection, and memory cells can stimulate a rapid immune response. Figure adapted from Wikimedia Commons.

The majority of people infected with SARS-CoV-2 develop specific antibodies ${ }^{3-5}$ and T-cell responses against the virus. ${ }^{6,7}$ Some studies suggest that the immune response varies by the clinical severity of infection. In a retrospective study of 21 adults with SARS-CoV-2 infection, Wang et al. demonstrated that there was a more pronounced increase in neutralizing antibodies after 3 weeks among 11 participants with severe infection compared with 10 participants with mild infection. ${ }^{3}$ Similarly, Long et al. performed a study comparing 37 symptomatic and 37 infected, but asymptomatic adults with confirmed SARS-CoV-2 infection. ${ }^{8}$ The authors showed that both groups developed an IgG antibody response to SARS-CoV-2, but the levels of IgG antibodies were higher in adults who were symptomatic compared to adults who were asymptomatic.

The duration of the antibody response also varies. In the study by Long et al., $81 \%$ of infected asymptomatic individuals and $62 \%$ of symptomatic individuals showed a decrease in the levels of neutralizing antibodies 8 weeks after hospital discharge. ${ }^{8}$ $40 \%$ of asymptomatic individuals did not have detectable antibody levels at this time point, compared with $13 \%$ of mildly symptomatic individuals. The decrease in circulating antibodies within weeks of SARS-CoV-2 infection is more rapid than after infection with the virus causing SARS or the virus causing MERS. Antibodies can still be detected for 1 year or longer following infection with these viruses. ${ }^{9-11}$ This led to concerns that those who have been infected with SARS-CoV-2 might not be immune to it. 


\section{Questions}

What is the clinical importance of the recently reported decrease in antibodies against SARS-CoV-2 over time?

What is the current role of serological testing for antibodies against SARS-CoV-2?

\section{Findings}

In the long term, the immune response to infection is mediated through memory $B$ cells, which produce antibodies against prior infection. In 41 adults who recovered from mild to moderate infection with SARS-CoV-2, there was evidence of memory $B$ cells in blood samples taken 1 month after their diagnosis. ${ }^{12}$ Furthermore, memory $B$ cells from the blood samples of recovered patients have also been shown to produce neutralizing antibodies against SARS-CoV-2, ${ }^{13,14}$ meaning memory B cells are likely to trigger a rapid production of specific antibodies upon a re-infection with SARS-CoV-2. Whether the memory B-cell response to SARS-CoV-2 infection varies between symptomatic and asymptomatic people remains unclear.

Adaptive immunity against SARS-CoV-2 also includes a T-cell response. ${ }^{6,15}$ Braun et al. found that 15 out of 18 COVID-19 patients (83\%) developed specific T cells against SARS-CoV-2, including memory T cells. ${ }^{15}$ These memory $T$ cells would likely stimulate a rapid cell-mediated immune response in case of a re-infection.

Finally, some people who have never been exposed to SARS-CoV-2 may have already acquired adaptive cellular immunity against the virus. Le Bert et al. frequently detected T-cell responses against SARS-CoV-2 in a group of 37 healthy individuals with no history of COVID-19 or contact with COVID-19 patients. ${ }^{6}$ However, T-cells from these healthy individuals tended to react to a different component of the virus than $T$ cells from a group of 36 COVID-19 patients. Other studies had similar findings. ${ }^{3,7,16,17}$ These $T$ cell responses are likely due to previous infections with other coronaviruses, such as those causing the common cold. Their clinical importance is unknown, but they could explain some of the extreme variation in severity of disease after SARS-CoV-2 infection, from asymptomatic to critically ill. ${ }^{17,18}$

Human data on whether and how infection with SARS-CoV-2 results in protective immunity against re-infection are lacking. However, a recent study in monkeys suggests that adaptive immunity against SARS-CoV-2 can help control the virus upon re-infection. ${ }^{19}$ When recovered monkeys were infected with SARS-CoV-2 for a second time, the peak viral loads in both the nose and the lungs were significantly lower as compared to the first time. The monkeys also developed a much stronger antibody response when re-infected. An extrapolation of these findings to humans suggests that adaptive immunity could also be protective in humans.

\section{Interpretation}

Antibody responses appear less pronounced in infected asymptomatic individuals than in symptomatic individuals, and antibody levels appear to decrease relatively fast after an infection with SARS-CoV-2, but the clinical importance of these observations is unknown.

The detection of both memory $T$ cells and memory $B$ cells from recovered individuals suggests the potential for the adaptive immune system to respond quickly upon a re-infection. Longitudinal studies are needed to elucidate if and how individuals who have already recovered from SARS-CoV-2 infection are protected from re-infections.

Due to the incomplete understanding of the role and kinetics of antibody response 
to SARS-CoV-2 at this time, testing for the presence of SARS-CoV-2 specific antibodies typically does not provide meaningful clinical information to the individual being tested. In general, serological testing should therefore be limited to research and public health use only. In specific clinical situations, such as the multisystem inflammatory syndrome in children, serological testing may have a clinical role. ${ }^{20}$

The adaptive immune response to SARS-CoV-2 discussed in this science brief only reflects what is observed in a natural infection. The vaccines currently being developed to fight against SARS-CoV-2 were designed to generate a stronger and more sustainable immune response than seen in a natural infection. The issues described in this science brief should not be used to predict the potential effects of future vaccines.

\section{Methods Used for This Science Brief}

We searched PubMed, Google Scholar, the COVID-19 Rapid Evidence Reviews, the Joanna Briggs Institute's COVID-19 Special Collection, LitCovid in PubMed, the Oxford COVID-19 Evidence Service, the World Health Organization's Global Literature on Coronavirus Disease, and other COVID-19 specific resources listed by the Guidelines International Network and the McMaster Health Forum. In addition, we retrieved reports citing relevant articles through Google Scholar and reviewed references from identified articles for additional studies. The search was last updated on August 8, 2020.

\section{Author Contributions}

TW, YC, AO and PJ wrote the first draft of the science brief. All authors contributed to the conception of the science brief, revised it critically for important intellectual content, and approved the final version.

\section{References}

1. Netea MG, Schlitzer A, Placek K, Joosten LAB, Schultze JL. Innate and Adaptive Immune Memory: an Evolutionary Continuum in the Host's Response to Pathogens. Cell Host Microbe. 2019;25(1):13-26. https://doi.org/10.1016/ j.chom.2018.12.006

2. Brynjolfsson SF, Persson Berg L, Olsen Ekerhult T, et al. Long-Lived Plasma Cells in Mice and Men. Front Immunol. 2018;9:2673. https://doi.org/10.3389/ fimmu.2018.02673

3. Wang $Y$, Zhang $L$, Sang $L$, et al. Kinetics of viral load and antibody response in relation to COVID-19 severity. J Clin Invest. Published online July 7, 2020. https://doi.org/10.1172/JCl138759

4. Robbiani DF, Gaebler C, Muecksch F, et al. Convergent antibody responses to SARS-CoV-2 in convalescent individuals. Nature. Published online June 18, 2020. https://doi.org/10.1038/s41586-020-2456-9

5. Schultheiß C, Paschold L, Simnica D, et al. Next-Generation Sequencing of $T$ and B Cell Receptor Repertoires from COVID-19 Patients Showed Signatures Associated with Severity of Disease. Immunity. Published online June 2020:S107476132030279X. https://doi.org/10.1016/j.immuni.2020.06.024

6. Le Bert N, Tan AT, Kunasegaran K, et al. SARS-CoV-2-specific T cell immunity in cases of COVID-19 and SARS, and uninfected controls. Nature. Published online 
July 15, 2020. https://doi.org/10.1038/s41586-020-2550-z

7. Grifoni A, Weiskopf D, Ramirez SI, et al. Targets of T Cell Responses to SARS-CoV2 Coronavirus in Humans with COVID-19 Disease and Unexposed Individuals. Cell. 2020;181(7):1489-1501.e15. https://doi.org/10.1016/j.cell.2020.05.015

8. Long Q-X, Tang X-J, Shi Q-L, et al. Clinical and immunological assessment of asymptomatic SARS-CoV-2 infections. Nat Med. Published online June 18, 2020:1-5. https://doi.org/10.1038/s41591-020-0965-6

9. Choe PG, Perera R a. PM, Park WB, et al. MERS-CoV Antibody Responses 1 Year after Symptom Onset, South Korea, 2015. Emerg Infect Dis. 2017;23(7):10791084. https://doi.org/10.3201/eid2307.170310

10. Cao W-C, Liu W, Zhang P-H, Zhang F, Richardus JH. Disappearance of antibodies to SARS-associated coronavirus after recovery. N Engl J Med. 2007;357(11):1162 -1163. https://doi.org/10.1056/NEJMc070348

11. Tang F, Quan Y, Xin Z-T, et al. Lack of peripheral memory B cell responses in recovered patients with severe acute respiratory syndrome: a six-year follow-up study. J Immunol. 2011;186(12):7264-7268. https://doi.org/10.4049/ jimmunol.0903490

12. Juno JA, Tan H-X, Lee WS, et al. Humoral and circulating follicular helper $\mathrm{T}$ cell responses in recovered patients with COVID-19. Nat Med. Published online July 13, 2020. https://doi.org/10.1038/s41591-020-0995-0

13. Zost SJ, Gilchuk P, Case JB, et al. Potently neutralizing and protective human antibodies against SARS-CoV-2. Nature. Published online July 15, 2020. https:// doi.org/10.1038/s41586-020-2548-6

14. Wan J, Xing S, Ding L, et al. Human-IgG-Neutralizing Monoclonal Antibodies Block the SARS-CoV-2 Infection. Cell Rep. 2020;32(3):107918. https:// doi.org/10.1016/j.celrep.2020.107918

15. Braun J, Loyal L, Frentsch M, et al. SARS-CoV-2-reactive T cells in healthy donors and patients with COVID-19. Nature. Published online July 29, 2020. https:// doi.org/10.1038/s41586-020-2598-9

16. Pinto D, Park Y-J, Beltramello $M$, et al. Cross-neutralization of SARS-CoV-2 by a human monoclonal SARS-CoV antibody. Nature. 2020;583(7815):290-295. https://doi.org/10.1038/s41586-020-2349-y

17. Mateus J, Grifoni A, Tarke A, et al. Selective and cross-reactive SARS-CoV-2 T cell epitopes in unexposed humans. Science. Published online August 4, 2020. https://doi.org/10.1126/science.abd3871

18. Sette A, Crotty S. Pre-existing immunity to SARS-CoV-2: the knowns and unknowns. Nat Rev Immunol. 2020;20(8):457-458. https://doi.org/10.1038/ s41577-020-0389-z

19. Chandrashekar A, Liu J, Martinot AJ, et al. SARS-CoV-2 infection protects against rechallenge in rhesus macaques. Science. Published online May 20, 2020:eabc4776. https://doi.org/10.1126/science.abc4776

20. Feldstein LR, Rose EB, Horwitz SM, et al. Multisystem Inflammatory Syndrome in U.S. Children and Adolescents. N Engl J Med. 2020;383(4):334-346. https:// doi.org/10.1056/NEJMoa2021680 